\title{
Cirugía laparoscópica avanzada robótica Da Vinci: origen, aplicación clínica actual en Urología y su comparación con la cirugía abierta y laparoscópica
}

\author{
H. Villavicencio Mavrich \\ Servicio de Urología. Fundació Puigvert. Barcelona. \\ Actas Urol Esp 2006; 30 (1): 1-12
}

\begin{abstract}
RESUMEN
CIRUGÍA LAPAROSCÓPICA AVANZADA ROBÓTICA DA VINCI: ORIGEN, APLICACIÓN CLÍNICA ACTUAL EN UROLOGÍA Y SU COMPARACIÓN CON LA CIRUGÍA ABIERTA Y LAPAROSCÓPICA

La cirugía abierta capaz de hacer grandes exéresis y reparaciones en tejidos frágiles, está siendo paulatinamente sustituida durante esta última década por la laparoscópica, por sus enormes beneficios para el paciente, sin olvidar las dificultades para su aprendizaje por los cirujanos que tienen que suplir estas deficiencias con mayor esfuerzo y estrés. La cirugía robótica viene a suplir las limitaciones de la cirugía laparoscópica convencional haciendo cirugías más ergonómicas y más precisas, sobre todo para aquellas cirugías más complejas y difíciles de acceder como es la prostatectomía radical. En esta revisión se hace un recordatorio de historia y aplicaciones clínicas de nuevas tecnologias avanzadas y robotizadas y en comparación con la cirugia abierta y la laparoscopia convencional.

Palabras clave: Cáncer de próstata. Prostatectomía, abierta, laparoscópica y robótica. Cirugía mínimamente invasiva. Da Vinci.

ABSTRACT

DA VINCI ADVANCED ROBOTIC LAPAROSCOPIC SURGERY: ORIGIN AND CURRENT CLINICAL APPLICATION IN UROLOGY, AND COMPARISON WITH OPEN AND LAPAROSCOPIC SURGERY

Over the last decade, open surgery, which is able to perform large extirpations and repairs of fragile tissues, is gradually being substituted with laparoscopic surgery due to the high benefits the latter entails for the patients, an also due to the learning difficulties for surgeons who must make up for such deficiencies applying higher efforts and a larger amount of stress. Robotic surgery stands in for the limitations of conventional laparoscopic surgery by means of performing more ergonomic and more accurate surgeries, particularly in the case of the most complex and difficult to access operations, such as radical prostatectomy. This review will perform a reminder of the history and clinical applications of new advanced and robotic technologies, and also a comparison with open surgery and conventional laparoscopy.
\end{abstract}

Keywords: Prostate cancer. Open, laparoscopic and robotic prostatectomy. Minimally invasive surgery. Da Vinci.

\section{$\mathrm{L}$} a informática se ha convertido en parte fundamental de la tarea clínica diaria y habitual. Actualmente siguen desarrollándose las nuevas disciplinas (p.ej. telecirugía, cirugía asistida por ordenador y por la robótica, cirugía guiada por imágenes, nanotecnología, teleconsultas médicas, teleseguimiento postoperatorio, etc.,) y no podemos menos que comenzar a preguntarnos cuáles serán las indicaciones futuras de la medicina asistida informáticamente. Lo que es cierto es que la revolución digital en medicina nos conduce al desarrollo de nuevas modalidades de tratamiento operatorio, simulaciones de cirugía y mejor enseñanza quirúrgica. 
En 1904 Halsted propuso una definición para "enseñanza quirúrgica». Si bien su trabajo era extremadamente detallista, el mensaje principal fue breve y significativo: "Ve una, realiza una y enseña una" ${ }^{1}$. De acuerdo con esta frase se introdujo en cirugía un término importante, la "curva de aprendizaje", que significa que cuanto menos experimentado sea un cirujano, más errores cometerá durante los procedimientos quirúrgicos. Según la regla de Halsted, para convertirse en un cirujano experto no hay más secreto que vencer la curva de aprendizaje, por lo que el proceso de formación quirúrgica es muy largo.

En la década de 1990, la cirugía laparoscópica modificó totalmente el estilo de las intervenciones quirúrgicas. A medida que avance el siglo XXI, esta modalidad está en demanda por parte de los pacientes que desean cirugía mínimamente invasiva por sus enormes ventajas con relación a la cirugía abierta (Tabla 1$)^{2}$.

Por otra parte, las técnicas complejas como la sutura y la ligadura, son dificiles para los cirujanos en el curso de la laparoscopia debido a la carencia del sentido del tacto, a la imagen bidimensional (2-D) que da el monitor, a la pérdida de coordinación de la mano (Fig.1) y la vista, puesto que los instrumentos han de moverse en la dirección opuesta de la que se desea en el monitor (es decir, el efecto de la imagen en el espejo) (Fig. 2) y de la limitación de las posibilidades motrices de la mano, la muñeca y los dedos. Todo esto hace que aumente la tensión mental y física a que está sometido el cirujano. Lo fundamental es que las intervenciones quirúrgicas han ido evolucionando a lo largo de los años sobre la base de los ojos entrenados y las manos hábiles del cirujano.

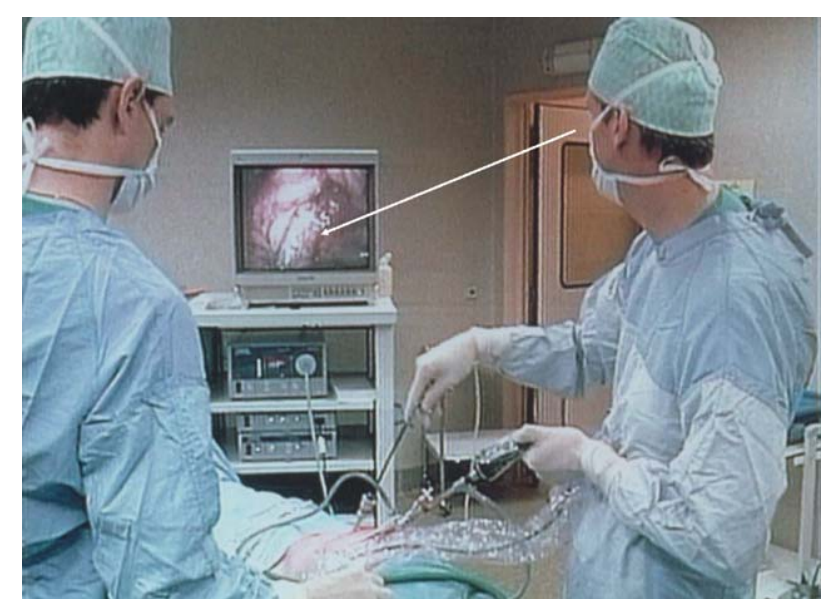

\section{FIGURA 1}

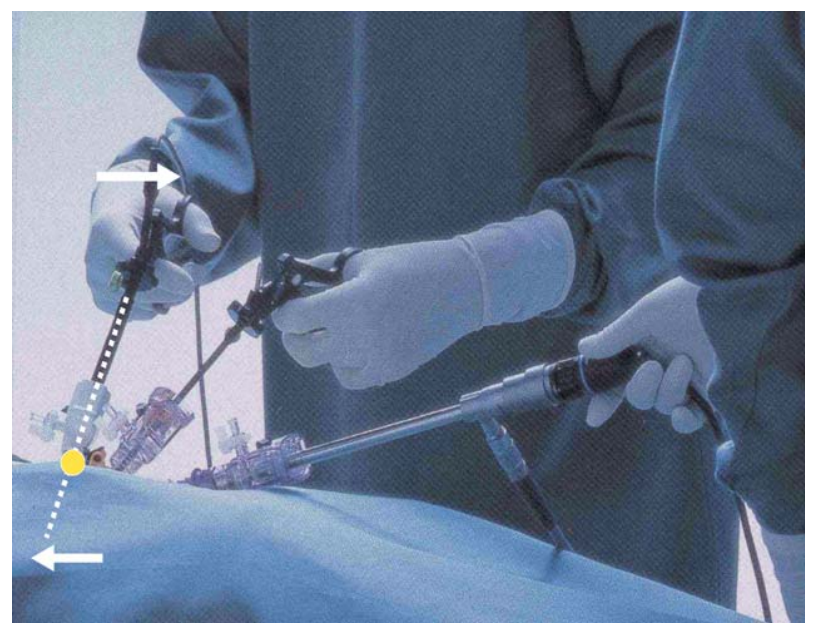

FIGURA 2

Estas limitaciones quedan superadas con las tecnologías informáticas avanzadas como los robots quirúrgicos y las imágenes tridimensionales (3-D). Los robots quirúrgicos como el Da Vinci® Surgical System (Intuitive Surgical, Mountain

\section{Tabla 1}

Ventajas y desventajas de la cirugía laparoscópica sobre la abierta

\section{Ventajas}

Menos anestesia/analgesia

Menor pérdida de sangre, dolor postoperatorio e incomodidad

Menor trauma para el organismo/menos formación de tejido cicatricial

Menor riesgo de infección

Hospitalización más corta

Recuperación/vuelta a las actividades diarias normales y al trabajo más rápida

\section{Desventajas}

Visión limitada del campo operatorio por pérdida de la visualización en 3-D

Pérdida de la coordinación manos-ojos

Habilidades motrices de manos, muñeca y dedos limitadas

Pérdida del sentido de profundidad

Suturas y ligaduras difíciles por rigidez de los instrumentos

La falta de ergonomía produce incomodidad, cansancio y estrés 
View, CA) proporcionan al cirujano técnicas manuales y visuales tecnológicamente avanzadas que han revolucionado muchos campos de la cirugía mínimamente invasiva ${ }^{3}$, superando claramente a la laparoscopia convencional sobre todo en cirugías delicadas y complejas al proporcionar al cirujano un mapa detallado para ver los tejidos sanos y para la preservación nerviosa, como es la prostatectomía radical (Fig. 3), por su excelente visión (3D), la maniobrabilidad de sus instrumentos articulados y la precisión quirúrgica, que contribuyen a realizar cirugias de mayor calidad y extraordinaria ergonomía.

\section{Los planos de la disección de la fascia son numerosos ¿cuáles son las opciones?}

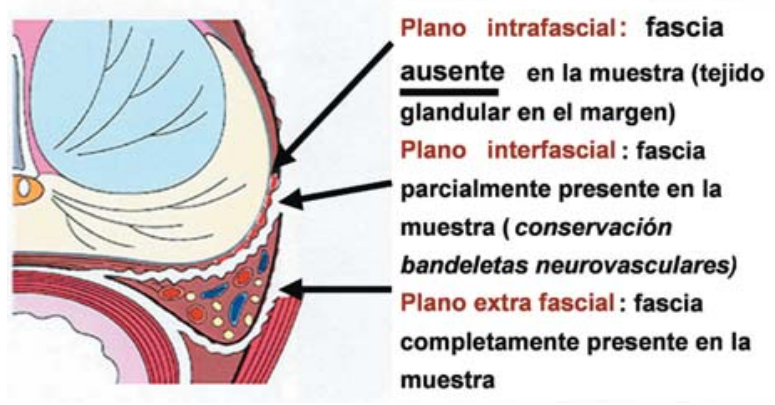

FIGURA 3

\section{El Da Vinci ${ }^{\circledR}$ Surgical System}

El Da Vinci® Surgical System es un sistema robótico asistido informáticamente (Fig. 4) que amplía la capacidad del cirujano de operar el interior del cuerpo humano de forma menos invasiva.

El sistema consta de tres partes principales:

1. La consola del cirujano, que está controlada por el mismo cirujano sentado en una posición cómoda y ergonómica (Figs. 5 y 6).

2. El Robot Quirúrgico, que se sitúa junto a la mesa de operaciones en la que está el paciente y del que salen dos brazos que realizan directamente el procedimiento (Fig. 7).

3. El sistema de visión, que es el tercer brazo del Robot Quirúrgico, sostiene una cámara endoscópica en 3-D de alta calidad (Fig. 7).

Un cuarto brazo puede emplearse para reemplazar a un asistente. La consola del cirujano consiste en un visualizador que presenta imáge-

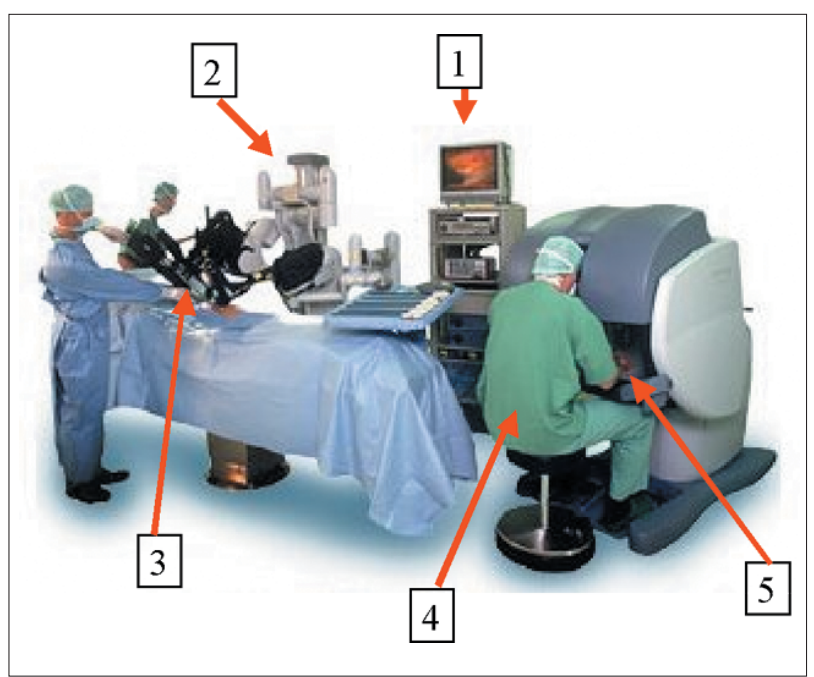

FIGURA 4: Da Vinci® Surgical System

1. Monitor del asistente.

2. Robot quirúrgico.

3. Los instrumentos intercambiables con tecnologia Endo-Wrist siguen al mismo tiempo los movimientos de la mano y la muñeca del cirujano.

4. El cirujano en la consola operatoria.

5. El cirujano aplica movimientos manuales de cirugia abierta, que los instrumentos duplican con precisión en el campo operatorio.

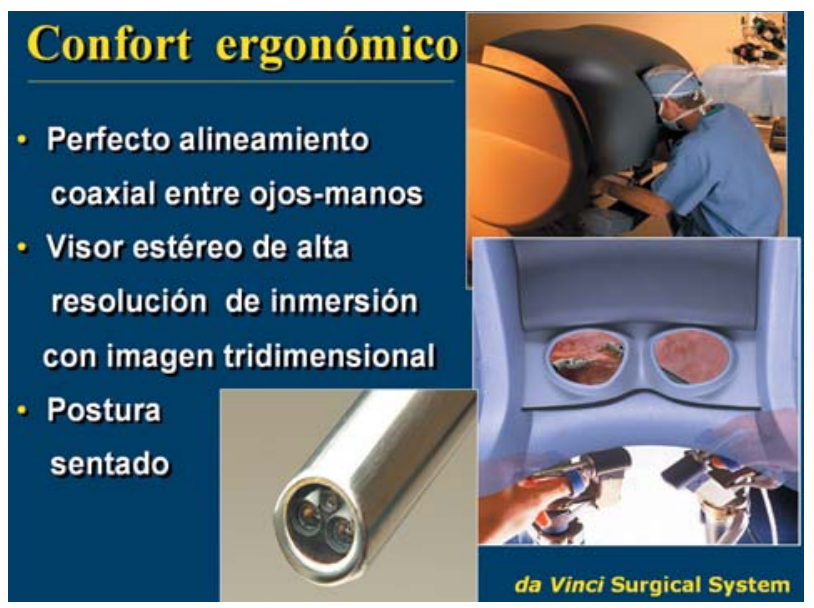

FIGURA 5

nes 3-D obtenidas a partir de la cámara endoscópica que está dentro del cuerpo del paciente. El término "manipulación amo-esclavo" se refiere a la consola del cirujano, equipada con manipuladores "amos" que controlan 1) los movimientos de los manipuladores quirúrgicos correspondientes ("manipulador esclavo") que sostienen los instrumentos quirúrgicos Endo Wrist ${ }^{\circledR}$ y 2) el manipulador de la cámara endoscópica durante el procedimiento. Este manipulador amo-esclavo permite 


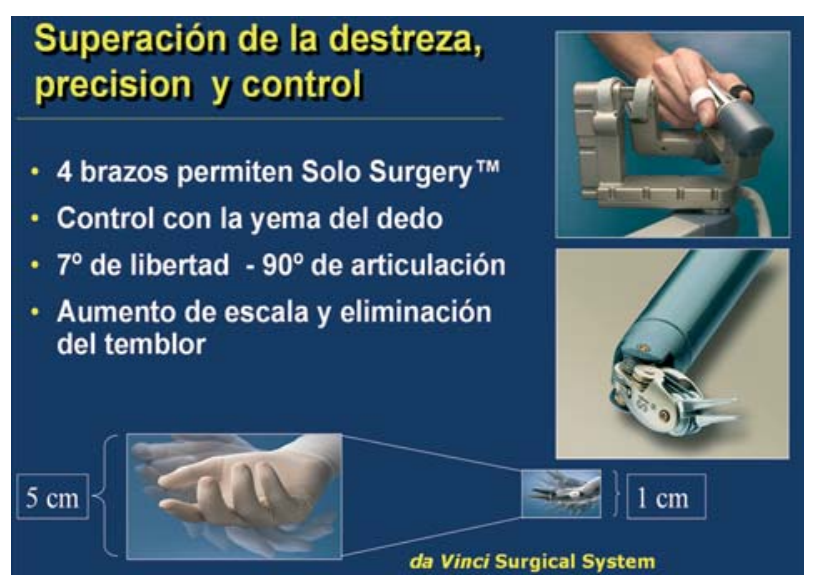

FIGURA 6

al cirujano la realización de procedimientos más precisos en comparación con la cirugía laparoscópica convencional. Los instrumentos Endo Wrist ${ }^{\circledR}$ (Fig. 8) otorgan al cirujano siete grados de libertad en comparación con los cuatro grados que proporciona la cirugía laparoscópica convencional y son comparables al movimiento de la mano humana en cuanto a aumento de la destreza.

Por lo tanto, el resultado es: 1) un traslado intuitivo del asa del instrumento al movimiento de la punta, eliminando así el efecto de imagen en el espejo y mejorando la coordinación mano-vista; 2) el escalado de los movimientos del instrumento (desde $1: 1$ a $1: 3$ y a $1: 5$ ), lo que permite una disección precisa y delicada (Fig. 9); 3) eliminación del temblor del cirujano gracias a los filtros informáticos, y 4) la alineación coaxial de los ojos, la mano y la imagen de la punta de la herramienta. Hasta ahora se han realizado muchos tipos de interven- ciones quirúrgicas con el sistema quirúrgico Da Vinci ${ }^{\circledR}$, en los campos abdominal, cardíaco, toracoscópico, ginecológico y urológico.

\section{Historia de la cirugia de cánceres asistida por robot (Da Vinci $\left.{ }^{\circledR}\right)$}

La Tabla 2 resume las utilidades del sistema quirúrgico Da Vinci® en distintos tipos de cirugía abdominal, cardíaca, toracoscópica, ginecológica y urológica.

\section{Cirugía abdominal}

Los primeros informes sobre el uso de laparoscopia asistida por la robótica, provienen del campo de la cirugía abdominal en el contexto de enfermedades gastrointestinales. Ya en 1997 se llevó a cabo una colecistectomía laparoscópica aplicando un prototipo del sistema quirúrgico Da Vinci ${ }^{4}$. En los años que siguieron, el mismo grupo notificó el empleo de este sistema laparoscópico telerrobotizado en un bypass gástrico ${ }^{5} \mathrm{y}$ una fundoplicación de Nissen ${ }^{6}$ pero también se realizaron con éxito una miotomía de $\mathrm{Heller}^{7}$, una desvascularización gástrica y una esplenectomía por hipertensión portal ${ }^{8}$. Además, la cirugía como tratamiento de enfermedades inflamatorias se adaptaba perfectamente al uso del sistema Da Vinci® (p.ej. colectomía sigmoidea por diverticu$\operatorname{losis}^{9}$ ). Más concretamente dentro del campo de la oncología gastrointestinal se informó sobre el uso de la máquina Da Vinci® en la primera gastrectomía distal laparoscópica totalmente intraabdominal debida a cáncer gástrico ${ }^{8,9}$. Melvin et al. ${ }^{10}$ describieron una resección pancreática por medio del sistema quirúrgico Da Vinci ${ }^{\circledR}$ en una
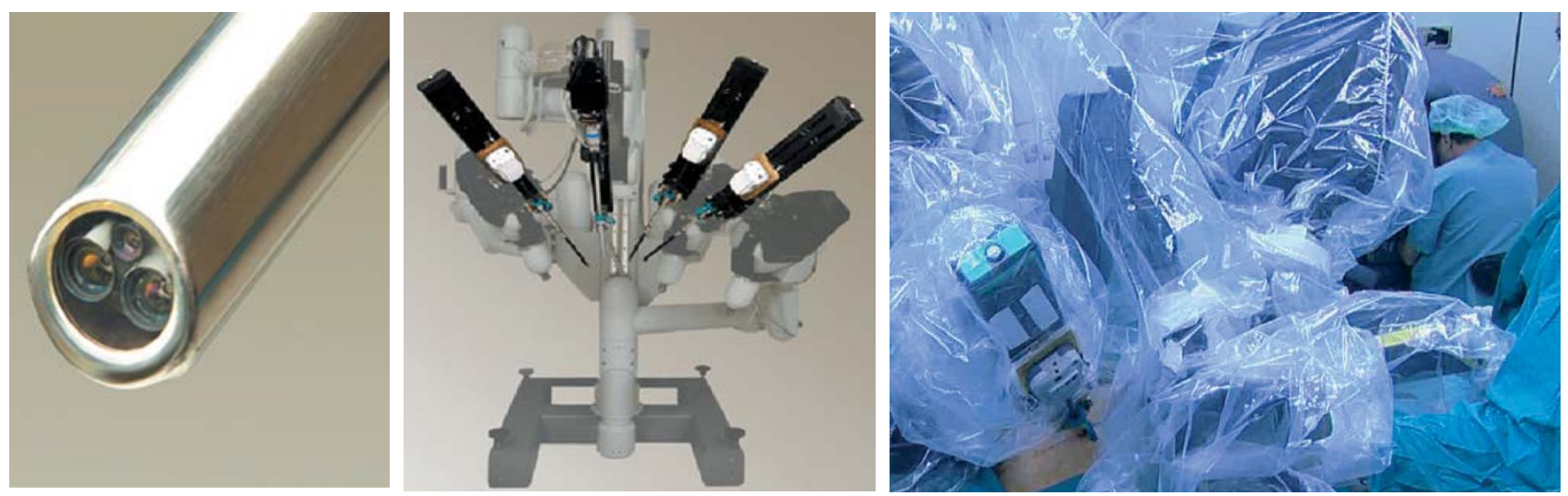

FIGURA 7 


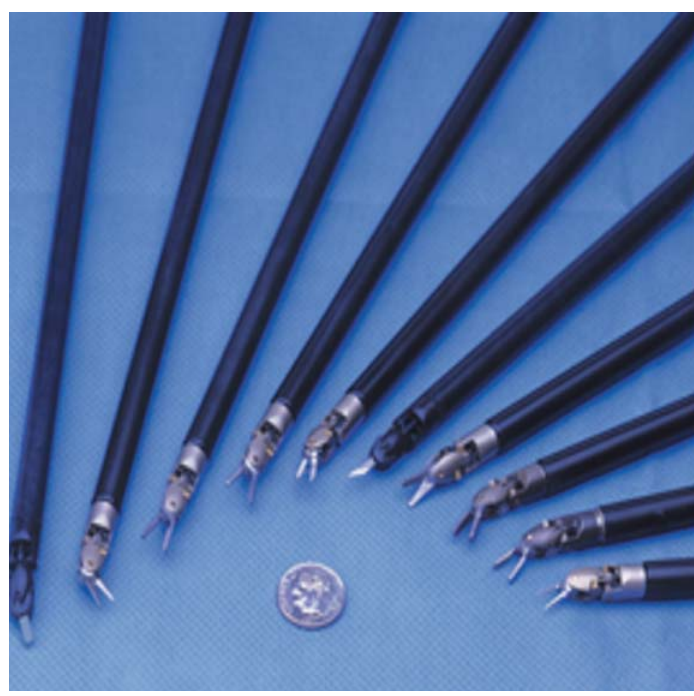

FIGURA 8: La tecnologia de la Endo Wrist@.

mujer que presentaba dolor de espalda como resultado de una masa quística compleja en la cola del páncreas.

\section{Cirugía ginecológica}

Otros procedimientos quirúrgicos abdominales en los que se aplicó el Da Vinci® entraban dentro del terreno ginecológico y el primer caso clínico de trasposición ovárica laparoscópica con ayuda robótica fue una recolocación anatómica de los ovarios de la pelvis en el abdomen en mujeres que esperaban recibir radiación pelviana y reanastomosis de las trompas de Falopio $^{11}$. Actualmente se utiliza también para ligadura de trompas, histerectomía y miomectomía; el uso del sistema quirúrgico $\mathrm{Da}$ Vinci® en estas dos últimas indicaciones quirúrgicas se acaba de aprobar por la FDA (abril de 2005).

\section{Cirugía cardíaca}

Uno de los campos en que la robótica está transformando drásticamente la medicina y que origina más entusiasmo, es la cirugía cardíaca mínimamente invasiva. El sistema Da Vinci ${ }^{\circledR}$ se diseñó expresamente para realizar bypass coronarios a tórax cerrado ${ }^{12}$. El resultado es que los cirujanos cardíacos han acumulado una gran cantidad de experiencia práctica en el uso de este sistema. En 1999 se describió el primer uso del Da Vinci ${ }^{\circledR}$ en un bypass coronario a tórax cerra$\mathrm{do}^{13}$ y sólo un año después se empleó para disecar las arterias mamarias internas tanto derecha como izquierda para realizar bypass coronario en 27 pacientes ${ }^{14}$. Mohr et al. llevaron a cabo cirugía de bypass coronario en 148 pacientes ${ }^{15}$. En suma, se utilizó el Da Vinci® para disecar 81 arterias mamarias internas izquierdas (AMII) y para suturar 15 injertos de AMII a bypasses de arteria descendente anterior $(\mathrm{ADA})$ por medio de una incisión de esternotomía media. Después de estos procedimientos se realizaron otros 27 bypasses de AMII a ADA en un corazón parado y a tórax cerrado. Más recientemente, el mismo grupo realizó con éxito el mismo procedimiento en un corazón funcionante y a tórax cerrado ${ }^{16}$. Al mismo tiempo, Kappert y Cichon ${ }^{17}$ realizaron 37 bypasses coronarios totalmente laparoscópicos prescindiendo de la bomba en corazones funcionantes con el sistema quirúrgico Da Vinci®. Prasad et al. ${ }^{18}$ construyeron con éxito anastomosis de la arteria torácica interna izquierda (ATII) a ADA en 19 pacientes. También se empleó con éxito el Da Vinci® para la reparación de válvulas mitrales ${ }^{19}$.

\section{Cirugía toracoscópica}

Asímismo se introdujo con éxito la cirugía mínimamente invasiva en el campo de la cirugía

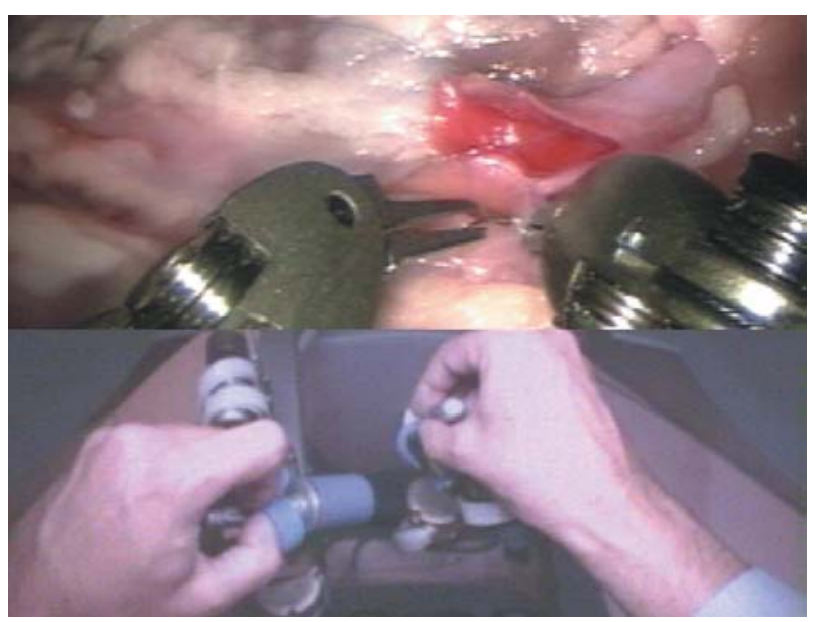

FIGURA 9 
Tabla 2

Tipos de cirugía en que se ha aplicado el Da Vinci® Surgical System

\begin{tabular}{|c|c|c|}
\hline $\begin{array}{l}\text { Sistema o } \\
\text { Aparato }\end{array}$ & Tipo de cirugia & $\begin{array}{c}\text { Aprobado } \\
\text { por FDA }\end{array}$ \\
\hline Abdominal & $\begin{array}{l}\text { Colecistectomía } \\
\text { Bypass gástrico } \\
\text { Fundoplicación de Nissen } \\
\text { Colectomía sigmoidea } \\
\text { Miectomía de Heller } \\
\text { Desvascularización gástrica/gastrectomía } \\
\text { Resección del páncreas }\end{array}$ & 2000 \\
\hline Cardíaco & $\begin{array}{l}\text { Reparación válvula mitral } \\
\text { Injerto con bypass de la arteria coronaria } \\
\text { LIMA a LAD (arterias mamarias internas } \\
\text { izquierdas a descendiente anterior izq. }\end{array}$ & 2002 \\
\hline Toracoscópico & $\begin{array}{l}\text { Lobectomias } \\
\text { Enucleaciones tumorales } \\
\text { Timomectomía post-timoma } \\
\text { Resección de Schwannoma }\end{array}$ & \\
\hline Ginecológico & $\begin{array}{l}\text { Ligadura de trompas / reanastomosis } \\
\text { Histerectomía } \\
\text { Miomectomía } \\
\text { Salpingo-ooforectomía } \\
\text { Trasposición ovárica }\end{array}$ & $\begin{array}{c}2003 \\
y \\
2004\end{array}$ \\
\hline Urológico & $\begin{array}{l}\text { Prostatectomía radical confinada } \\
\text { al órgano } \\
\text { Adrenalectomía } \\
\text { Trasplante renal / nefrectomía del donante }\end{array}$ & 2001 \\
\hline
\end{tabular}

nica y su posterior estandarización en el Hospital Henry Ford (Detroit, Michigan, USA) Al año siguiente se realizaron con Da Vinci ${ }^{\circledR}$ tanto una adrenalectomía por incidentaloma suprarrenal $^{26}$ como una nefrectomía de donante para un trasplante de riñón ${ }^{27,28}$. En la actualidad, esta lista ya ha sido ampliamente superada (ureteropieloplastia, cistectomía radical con neovejiga, suspensión de cuello vesical, sacrocolpopexia, nefrectomía parcial, etc).

\section{Laparoscopia con asistencia robótica frente a laparoscopia convencional y a cirugia abierta en la prostatectomia radical}

Prostatectomía radical retropúbica

abierta frente a prostatectomía laparoscópica

La prostatectomía radical retropúbica abierta (PRR) es una técnica bien arraigada y que se considera sin lugar a dudas el patrón oro para el tratamiento quirúrgico del cáncer de próstata confinado al órgano ${ }^{29-32}$ con

toracoscópica. Melfi y colegas $^{20}$ llevaron a cabo varias operaciones toracoscópicas (lobectomía, enucleaciones tumorales, extirpaciones y sutura de ampollas finalizado con pegamento de fibrina en neumotórax espontáneos) y utilizaron el sistema Da Vinci® en 12 casos. Además de la cirugía relacionada con el aparato respiratorio, se hizo una timomectomía toracoscópica en un paciente varón de 74 años que mostraba timoma ${ }^{21}$ y una resección de un schwannoma a una mujer de 46 años que presentaba una masa paravertebral izquierda en el tórax ${ }^{22}$ y en ambos casos se usó Da Vinci®.

\section{Cirugía Urológica}

Finalmente, la cirugía urológica minimamente invasiva se basa en los trabajos pioneros de Abbou, Binder, Pasticier y Menon, quiénes en 2001 introdujeron un abordaje anatómico de la prostatectomía radical con asistencia robótica ${ }^{23-25}$. Este último es considerado el diseñador de la téc- sus ventajas y sus inconvenientes (Tabla 3). En 1982, Walsh ${ }^{33}$ estableció las bases de la PRR anatómica contemporánea sobre la base de su obra anterior que delineaba la anatomía del complejo venoso dorsal y los nervios cavernosos ${ }^{34}$. Como ya se ha dicho, la aceptación de los procedimientos quirúrgicos por parte del paciente aumenta a medida que va perfeccionando la cirugía minimamente invasiva. Debido a ello, los urólogos comenzaron a elaborar técnicas menos invasivas como alternativa a la PRR. Para que esas técnicas se adoptasen, debían proporcionar resultados oncológicos y funcionales por lo menos equivalentes a los de la PRR normal.

El empleo de la prostatectomía radical laparoscópica (PRL) como alternativa mínimamente invasiva a la PRR abierta fue descrito por primera vez por Schuessler en 199735. Guillonneau y Valancien $^{36,37}$ de la clínica francesa Montsouris, popularizaron la PRL y lo mismo hicieron algunos centros especializados de Europa en los años 
Tabla 3

Cirugía abierta

\begin{tabular}{ll}
\hline Ventajas & Inconvenientes \\
Estandarizadas & $\begin{array}{l}\text { Técnicas traumáticas para el } \\
\text { paciente } \\
\text { Con riesgo para los cirujanos } \\
\text { (pinchazos) }\end{array}$ \\
Fáciles de enseñar y & $\begin{array}{l}\text { Mucha medicación para mitigar el } \\
\text { dolor }\end{array}$ \\
controlar & $\begin{array}{l}\text { Estancias hospitalarias y } \\
\text { convalecencia prolongadas }\end{array}$ \\
\hline
\end{tabular}

siguientes $^{37-39}$. En un intento de caracterizar el desenlace patológico de ambas técnicas, Brown et al. ${ }^{40}$ compararon los índices de márgenes positivos en muestras de patología obtenidas por PRR y por PRL. De las series de PRL ${ }^{36-41}$ llevadas a cabo en Europa se obtuvieron índices de márgenes positivos del $11 \%$ al 28\%. Es probable que la variabilidad de estos indices positivos refleje un sesgo de la selección inicial porque a menudo, al embarcarse en este procedimiento técnicamente exigente, los cirujanos eligen los pacientes que consideran ideales. Brown et al. ${ }^{40}$ neutralizaron este sesgo al analizar 60 pacientes consecutivos con PRL y comparar sus hallazgos patológicos con los de 60 pacientes consecutivos con PRR y una cohorte de 60 pacientes con PRR con grado y estadio clínico similares según la biopsia transrectal.

Independientemente del acceso quirúrgico utilizado, no se observaron índices de márgenes positivos estadísticamente diferentes ( $p>0,1)$ (PRL: 16,9\% vs. PRR: 20\%) si bien, el grupo de la PRR tuvo un índice más bajo de márgenes positivos en el ápex y en sitios múltiples. La pérdida de sangre y las tasas de transfusión fueron mucho menores con el acceso laparoscópico. En un pequeño estudio que comparaba la PRR con la PRL, se evaluaron los resultados funcionales (continencia y potencia) un año después de la cirugía ${ }^{42}$. Al inicio, antes de la cirugía, de 300 pacientes (70 con PRR y 230 con PRL), el 98,7\% estaban continentes y el $96,6 \%$ conservaban la poten- cia. Las dos técnicas exhibieron resultados similares (Tabla 4) con respecto a la continencia y a la función eréctil al año de la cirugía. Si embargo, la recuperación de la continencia nocturna pareció tener lugar más temprano en los casos de PRL. De acuerdo con estos resultados, se puede concluir que la PRL en manos de cirujanos expertos es segura y proporciona resultados oncológicos y funcionales equivalentes a los de la técnica abierta. La PRL se compara favorablemente con la PRR en términos de pérdida de sangre, hospitalización y tiempo de sondaje y actividad laboral.

Sin embargo, el mayor inconveniente de la PRL es su dificultad técnica $y$, por ende, su curva de aprendizaje pronunciada y prolongada, llegando en algunas series a una alta tasa de reconversión inicial ${ }^{42 \text { bis }}$. Probablemente se necesiten entre 40 y 100 procedimientos antes de llegar a ser competente en esta operación ${ }^{43}$ e incluso, una vez dominada la curva de aprendizaje, el tiempo operatorio sigue siendo más largo con la PRL (aproximadamente entre 3 y 4,5 horas) que con la PRR. Esto es inaceptable para muchos cirujanos "abiertos» que trabajan en consultas privadas o en un hospital no universitario. En un estudio reciente ${ }^{43}$ un urólogo principiante en la técnica laparoscópica que trabajaba en un hospital no universitario, siguió la curva de aprendizaje aplicando la PRL extraperitoneal en vez de la transperitoneal ${ }^{44}$. Los resultados mostraron una correlación significativa ( $\mathrm{p}<0,001$, Fig. 10) entre la progresión del número de casos y la duración (media de 160 minutos o 2,7 horas) de la cirugía.

El margen quirúrgico positivo fue del 17\%. En sólo el 14\% de los pacientes se presentaron complicaciones de menor importancia, que se carac-

\section{Tabla 4}

Prostatectomía radical retropúbica versus prostatectomía radical laparoscópica: resultados funcionales ${ }^{42}$

\begin{tabular}{lccc}
\hline & PRR & PRL & Valor de P \\
\hline Continencia diurna & $66,7 \%$ & $71,6 \%$ & $\mathrm{p}<0,05$ \\
Continencia nocturna & $66,7 \%$ & $87,1 \%$ & $\mathrm{p}=0,008$ \\
Potencia & $30 \%$ & $41 \%$ & $\mathrm{p}<0,05$ \\
$\quad$ Sin conservación neurovascular & $30 \%$ & $41 \%$ & $\mathrm{p}<0,05$ \\
Conservación neurovascular unilateral & $27 \%$ & $46 \%$ & $\mathrm{p}<0,05$ \\
Conservación neurovascular bilateral & $44 \%$ & $53 \%$ & $\mathrm{p}<0,05$ \\
\hline
\end{tabular}




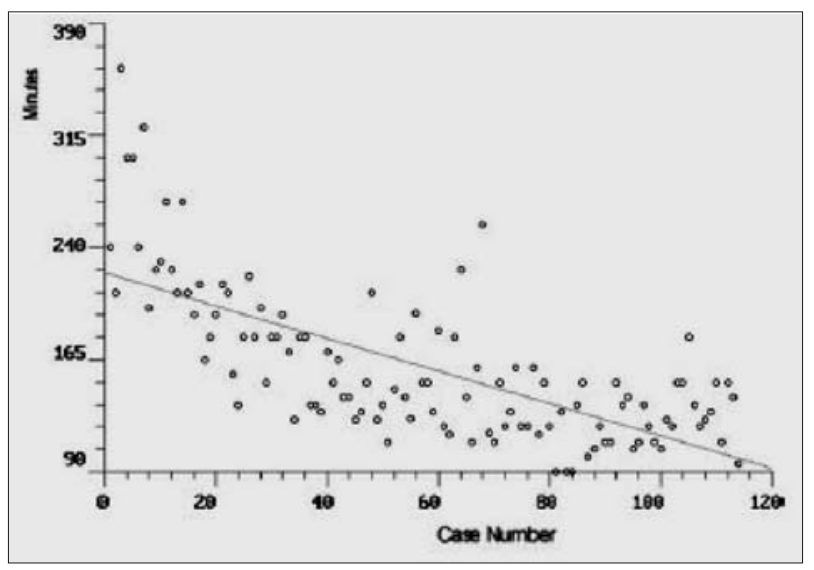

FIGURA 10: El tiempo operatorio disminuye a medida que aumenta el número de PRL extraperitoneales realizadas $^{43}$.

terizaron por aparición precoz (50\%) o tardía (59\%). En todas las complicaciones precoces fue innecesaria una segunda operación, en tanto que las tardías se trataron con éxito endoscópicamente. Con respecto a los tiempos operatorios informados, debe mencionarse que el objetivo primordial de la operación, según se afirmó en las entrevistas previas al procedimiento, era el control del cáncer y por lo tanto no se efectuó conservación bilateral de los nervios; solamente en el $21 \%$ de los pacientes se valoró la conservación unilateral de los nervios. En general, después de una formación racional del cirujano durante un periodo preliminar de seis meses, el urólogo que está comenzando a practicar la laparoscopia puede empezar a realizar la PRL con seguridad y pocas complicaciones y con tiempos operatorios comparables a los de la cirugía abierta.

Prostatectomía radical retropúbica abierta frente a laparoscópica con asistencia robótica

Otra manera de abreviar la curva de aprendizaje laparoscópico (para los cirujanos abiertos experimentados) y de disminuir el tiempo operatorio a la vez que se mantienen los beneficios de la laparoscopia minimamente invasiva, es aplicar la prostatectomía laparoscópica asistida por robot Da Vinci (PdV) (Figura 11) ${ }^{45,46}$. La PdV también ha demostrado ser más cómoda para el cirujano mientras opera al paciente y se acerca más a la cirugía abierta porque permite aplicar una enorme precisión y control al realizar las incisiones en los tejidos y al colocar las suturas exactas, debido a la excelente visión 3-D y a los movimientos de sus instrumentos articulados.

Algunos centros han publicado resultados en los que la PdV se comparaba con la PRR y que comprendía una considerable cantidad de pacientes. En un estudio comparativo y no aleatorizado llevado a cabo en un solo centro ${ }^{46}$ que incluía a 300 pacientes (100 PRR y 200 PdV) del Instituto Vattikuti de los Estados Unidos (instituto que comenzó a realizar PdV en el 2000), las mediciones de los resultados fueron similares con ambas técnicas.

La duración media de la operación (desde la incisión o disección hasta el cierre) también fue similar en la PRR (163 min y en la PdV (160 min) (Tabla 5).

Por el contrario, la pérdida estimada de sangre difirió de forma significativa $(\mathrm{p}<0,001)$ y posteriormente la necesidad de transfusión de sangre fue mayor en el grupo de la PRR (67\% vs) ninguno, $\mathrm{p}<0,001)$. También la puntuación del dolor (utilizando una escala visual analógica) fue significativamente mayor $(\mathrm{p}=0,05)$ en el grupo de la PRR cuando se lo comparó con los pacientes tratados por medio de PdV. La estancia hospitalaria media fue más larga para los pacientes tratados por PRR $(\mathrm{p}<0,05)$, mientras el 93\% de los pacien-

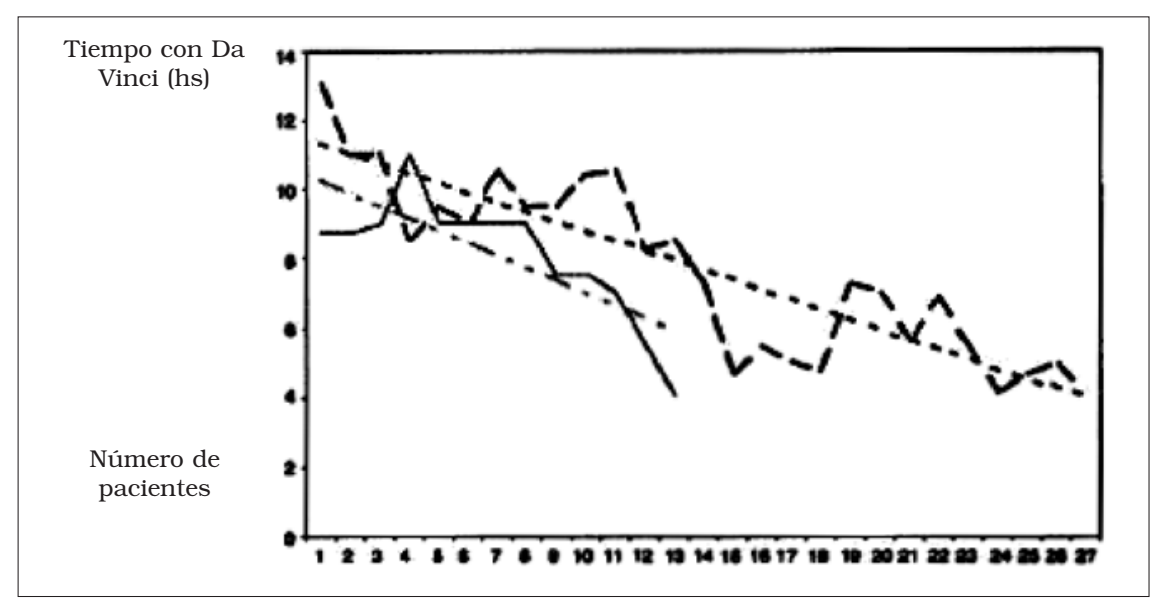

FIGURA 11: Curvas de aprendizaje. Tiempo operatorio de dos urólogos para un procedimiento laparoscópico, es decir, tiempo del sistema Da Vinci ${ }^{47}$. 
Tabla 5

PdV vs. PRR en 300 pacientes operados en el Inst. Vattikuti ${ }^{46}$

\begin{tabular}{lcc}
\hline & PRR (N = 100) & PdV (N = 200) \\
\hline Indice de masa corporal & 28 & 28 \\
Puntuación Gleason preoperatoria & 6,6 & 6,5 \\
Tiempo operatorio (horas) & 2,7 & 2,7 \\
Márgenes positivos (\%) & $23 \%$ & $9 \%$ \\
Pérdida de sangre (ml) & 910 & $153^{*}$ \\
Tasa de transfusiones (\%) & $11 \%$ & $0 \%{ }^{*}$ \\
VAS postoperatoria & 7 & $3^{*}$ \\
Estancia en el hospital (días) & 3,5 & $1,2^{*}$ \\
Tiempo de sondaje (dias) & 15,8 & $7^{*}$ \\
Complicaciones & $20 \%$ & $5 \%{ }^{*}$ \\
Tiempo mediano hasta continencia (dias) & 160 & $44^{*}$ \\
Tiempo mediano hasta que el 50\% de los & & \\
pacientes mantuvieron relaciones sexuales & & $340^{*}$ \\
(dias) & & \\
\hline
\end{tabular}

$* \mathrm{p}<0,05$

tes tratados con PdV se dieron de alta dentro de las $24 \mathrm{~h}$ frente a ninguno de los del grupo de PRR ( $\mathrm{p}<0,001)$.

Con respecto a la medición de resultados postoperatorios, los pacientes tratados por PdV demostraron tener cuatro veces menos complicaciones (Tabla 6, PRR: 20\%; PdV: 5\%; $\mathrm{p}<0,05)$, alcanzaron la continencia mucho antes (PRR: 440 días; PdV: 160 días; $\mathrm{p}<0,05)$ y por lo que se refiere a la función sexual, el 50\% de recuperación de la función eréctil (PRR: 440 días; PdV: 180 días, $\mathrm{p}<0,05)$ y de las relaciones sexuales (PRR: $>700$ días; PdV: 340 días, $\mathrm{p}<0,05$ ) pareció favorecer al grupo de la PdV.

Un inconveniente de este estudio comparativo es que mientras un equipo llevaba a cabo las PdV con exclusividad, en la misma institución ocho cirujanos diferentes se ocupaban de las PRR. Si bien esto plantea una inquietud válida, no afectó los resultados puesto que todos los cirujanos habían hecho al menos 100 PRR y tenían, entre todos, experiencia con más de 1.400 casos.

\section{Tabla 6} en la misma época ${ }^{46}$
Pero sí quedó flotando la pregunta de cómo se las arreglaría un solo cirujano con las dos técni$\operatorname{cas}^{48}$. A efectos prácticos, utilizar la propia experiencia como control interno minimiza los sesgos de la formación, la experiencia y la habilidad. Un cirujano experimentado de California, Ahlering, comparó los resultados de sus últimas 60 PRR con 60 PdV (después de haber remontado la curva de aprendizaje de 45

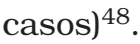

En general, los grupos de PdV y de PRR tuvieron (además de menos pérdida de sangre y hospitalizaciones más cortas con la PdV) resultados clínicos semejantes, entre ellos el tiempo operatorio, el estadio patológico del tumor, el índice de márgenes positivos y el porcentaje de complicaciones postoperatorias (Tabla 7).

A comparación similar llegaron diferentes cirujanos laparoscópicos convencionales ${ }^{39,42}$ que informaron de los mismos hallazgos entre la prostatectomía laparoscópica y la prostatectomía retropúbica abierta. Sin embargo, Tewari y asociados $^{46}$ notificaron mejores resultados tanto funcionales como oncológicos de la técnica robótica. Lo más probable es que la diferencia de resulta-

Complicaciones intra y postoperatorias (n) después de operar a 300 pacientes

\begin{tabular}{lccc}
\hline Complicaciones & PRR & PdV & Valor de P \\
\hline Operación abortada & 1 & 2 & NS \\
Conversión & - & 0 & NS \\
Lesiones rectales & 1 & 0 & NS \\
Illeo postoperatorio & 3 & 3 & NS \\
Dehiscencia/hernia de la herida & 1 & 2 & NS \\
Fiebre/neumonia postoperatoria & 4 & 0 & $<0,05$ \\
Linfocele & 2 & 0 & NS \\
Neuropatia obturadora & 2 & 0 & NS \\
Trombosis venosa profunda & 1 & 1 & NS \\
Infarto de miocardio postoperatorio & 1 & 0 & NS \\
Hemorragia postoperatoria & 4 & 1 & NS \\
\hline Total & $\mathbf{2 0}$ & $\mathbf{5}$ & $<\mathbf{0 5}$
\end{tabular}


Tabla 7

PdV vs PRR en 120 pacientes operados en la Universidad de California, Irvine ${ }^{46}$

\begin{tabular}{lcc}
\hline & $\begin{array}{c}\text { PRR } \\
\text { (N = 60) }\end{array}$ & $\begin{array}{c}\text { PdV } \\
\text { (N = 60) }\end{array}$ \\
\hline Índice de masa corporal & 27 & 26 \\
Tiempo operatorio (horas) & 3,6 & 3,9 \\
Márgenes positivos (\%) & $12 \%$ & $10 \%$ \\
Pérdida de sangre (ml) & 418 & $103^{*}$ \\
Tasa de transfusiones (\%) & $1 \%$ & $0 \%$ \\
Estancia en el hospital (días) & 2,2 & $1,1 *$ \\
Tiempo de sondaje (días) & 9 & 7 \\
Complicaciones & $10 \%$ & $6,7 \%$ \\
Continencia a los 3 meses & $75 \%$ & $76 \%$ \\
\hline
\end{tabular}

$* \mathrm{p}<0,05$

dos se refiriera a la excelencia de la robótica. No obstante, un hecho tangible y evidente es que los avances tecnológicos robotizados de los equipos, al aumentar el confort ergonómico de los cirujanos, la visión tridimensional y magnificada de sus lentes, la movilidad de sus instrumentos miniaturizados y la ausencia de temblor, hace que los rendimientos sean mayores para los cirujanos y, a la vez, las cirugías sean más precisas para los pacientes.

El uso del Robot Da Vinci en los Estados Unidos y en Europa aumenta de año en año. En tanto que en 2003 se realizaron más de 2.500 procedimientos, en 2004 la cifra aumentó hasta los 8.400. En Europa el empleo de la PdV es bastante menor que en los EE.UU. En enero de 2005 habian 70 robots Da Vinci ${ }^{\circledR}$ en toda Europa, en comparación con los 260 de los EE.UU. El centro más activo de Europa, el Karolinska Hospital de Estocolmo, realizó 204 PdV frente a 95 PRR en 2004. Indudablemente un cirujano abierto con mucha experiencia es capaz de dominar la curva de aprendizaje de "menos 3 horas" dentro de los 6 casos. En Europa existen sólo cinco centros que utilizan el robot Da Vinci exclusivamente para Urología (Tabla 8). En España, La Fundació Puigvert fue el primer Centro en incorporar esta tecnología y la primera intervención que se realizó fue precisamente urológica, concretamente una prostatectomía radical, practicada por el propio autor de este artículo ${ }^{49}$.
Tabla 8

Centros europeos que se dedican sólo a la Urología

\begin{tabular}{cc}
\hline Karolinska & Estocolmo \\
Hirslanden & Zurich \\
Gay's Hospital & Londres \\
St. Augustin & Burdeos \\
Fundació Puigvert & Barcelona \\
\hline
\end{tabular}

\section{¿Desde aquí hasta dónde?}

Aún cuando la cirugía con asistencia robótica ya ha revolucionado la profesión quirúrgica, siguen sin resolverse varios problemas fundamentales que impiden que se extienda más rápidamente y de forma más generalizada. Uno de los temas principales es el precio de los sistemas robóticos y su cobertura por el seguro social. Desde luego, en vista del éxito de la cirugía laparoscópica durante los últimos 10 años, no queda duda de que se estimulará el empleo de cirugía con asistencia robótica.

El robot Da Vinci, tiene una gran ventaja para los cirujanos expertos en cirugía abierta, no precisan aprendizaje previo ya que los conocimientos en cirugia abierta son fielmente imitados. Los movimientos de los dedos del cirujano son reproducidos a la perfección por los instrumentos miniaturizados y articulados de los brazos del robot. Para efectuar cirugía robótica en pacientes, sólo se requieren intervenciones previas en cadáveres y supervisión de las primeras intervenciones por un experto ${ }^{49}$. Las cirugias abiertas serán paulatinamente sustituídas por la laparoscopia convencional y esta tecnología se convertirá en un proceso de transición hacia la cirugía robótica ${ }^{49}$. Con la robótica se optimiza la ergonomía del cirujano, se disminuye drásticamente el estrés y el cansancio gracias a la calidad de la imagen tridimensional, la perfecta sincronización manos-ojos, la precisión de sus instrumentos articulados, la exactitud de sus suturas y la falta de temblor del cirujano y la excelente percepción de profundidad, hacen que los pacientes tengan unas cirugías más precisas y perfectas.

Las técnicas de cirugía con asistencia robótica también pueden beneficiar a un espectro mucho más amplio de investigadores en ingeniería tanto académica como industrial. 
Con respecto a las aplicaciones clínicas, existen seguidores y detractores, pero, en definitiva, imparable su uso en el futuro inmediato, puesto que casi toda la cirugía se realizará con robots ${ }^{49}$. Pero para que ello ocurra es necesario alcanzar: 1) mejorar el precio de los robots quirúrgicos y 2) la reducción de su tamaño.

Otro campo de los esfuerzos de la investigación son las aplicaciones de la microrrobótica ${ }^{50}$. La miniaturización de los robots tiene aplicaciones atractivas, entre ellas la de dispositivos que sean capaces de navegar de forma independiente por el sistema gastrointestinal, urinario o sanguíneo.

\section{CONCLUSIONES}

La cirugía robótica es un avance técnico mínimamente invasivo que está fascinando a los cirujanos. El uso de robots como el Da Vinci® indudablemente ha colocado al futuro de la cirugía urológica en situación de velocidad multiplicada, ha eliminado los principales inconvenientes de la laparoscopia convencional: la visualización, la coordinación manos-ojos y las limitaciones del movimiento manual. Estos avances han hecho disminuir la curva de aprendizaje y el tiempo operatorio y la convierten en un procedimiento quirúrgico atractivo e inimaginable para los cirujanos.

De momento, el paciente está recibiendo los mismos beneficios de la prostatectomía abierta y de la laparoscópica, comparables en eficacia en cuanto a parámetros clínicos y oncológicos pero optimizados en confort para los cirujanos y precisión en las cirugias para los pacientes con incisiones mínimas, menos anestesia, menor pérdida de sangre, hospitalizaciones más cortas $\mathrm{y}$, como consecuencia, retorno más rápido a las actividades laborales, lo que traduce un impacto positivo en la calidad de vida de los pacientes. Es de esperar que las mejoras tecnológicas den otro paso de gigante en el futuro y desarrollen una nueva generación de robots y de instrumentos que probablemente por su perfección puedan sustituir a los cirujanos actuales... que esto tarde en llegar.

\section{REFERENCIAS}

1. Grillo HC. To impart this art: the development of graduate surgical education in the United States. Surgery 1999;125 (1): $1-14$.

2. Lanfranco AR, Castellanos AE, Desai JP, Meyers WC. Robotic surgery: a current perspective. Ann Surg 2004;239 (1):14-21.
3. Hashizume M, Konishi K, Tsutsumi N, Yamaguchi S, Shimabukuro R. A new era of robotic surgery assisted by a computer-enhanced surgical system. Surgery 2002;131 (1 Suppl):S330-S333.

4. Himpens J, Leman G, Cadiere GB. Telesurgical laparoscopic cholecystectomy. Surg Endosc 1998;12(8): 1091.

5. Cadiere GB, Himpens J, Vertruyen M, Favretti F. The world's first obesity surgery performed by a surgeon at a distance. Obes Surg 1999;9(2):206-209.

6. Cadiere GB, Himpens J, Vertruyen M, Bruyns J, Fourtanier G. [Nissen fundoplication done by remotely controlled robotic technique]. Ann Chir 1999;53(2):137-141.

7. Melvin WS, Needleman BJ, Krause KR, Wolf RK, Michler $\mathrm{RE}$, Ellison EC. Computer-assisted robotic heller myotomy: initial case report. J Laparoendosc Adv Surg Tech A 2001; 11(4):251-253.

8. Hashizume M, Shimada M, Tomikawa M, Ikeda Y, Takahashi I, Abe R, et al. Early experiences of endoscopic procedures in general surgery assisted by a computerenhanced surgical system. Surg Endosc 2002;16(8):11871191.

9. Weber PA, Merola S, Wasielewski A, Ballantyne GH. Telerobotic-assisted laparoscopic right and sigmoid colectomies for benign disease. Dis Colon Rectum 2002;45(12): 1689-1694.

10. Melvin WS, Needleman BJ, Krause KR, Ellison EC. Robotic resection of pancreatic neuroendocrine tumor. J Laparoendosc Adv Surg Tech A 2003;13(1):33-36.

11. Degueldre M, Vandromme J, Huong PT, Cadiere GB. Robotically assisted laparoscopic microsurgical tubal reanastomosis: a feasibility study. Fertil Steril 2000;74(5): 1020-1023.

12. Falk V, Diegler A, Walther T, Autschbach R, Mohr FW. Developments in robotic cardiac surgery. Curr Opin Cardiol 2000;15(6):378-387.

13. Carpentier A, Loulmet D, Aupecle B, Berrebi A, Relland J. Computer-assisted cardiac surgery. Lancet 1999;353 (9150):379-380.

14. Kappert U, Cichon R, Gulielmos V, Schneider J, Schramm I, Nicolai J, et al. Robotic-enhanced Dresden technique for minimally invasive bilateral internal mammary artery grafting. Heart Surg Forum 2000;3(4):319-321.

15. Mohr FW, Falk V, Diegeler A, Walther T, Gummert JF, Bucerius J, et al. Computer-enhanced "robotic" cardiac surgery: experience in 148 patients. J Thorac Cardiovasc Surg 2001;121(5):842-853.

16. Falk V, Walther T, Stein H, Jacobs S, Walther C, Rastan A, et al. Facilitated endoscopic beating heart coronary artery bypass grafting using a magnetic coupling device. J Thorac Cardiovasc Surg 2003;126(5):1575-1579.

17. Kappert U, Cichon R, Schneider J, Gulielmos V, Ahmadzade T, Nicolai $J$, et al. Technique of closed chest coronary artery surgery on the beating heart. Eur $\mathrm{J}$ Cardiothorac Surg 2001;20(4):765-769.

18. Prasad SM, Ducko CT, Stephenson ER, Chambers CE, Damiano RJ, Jr. Prospective clinical trial of robotically assisted endoscopic coronary grafting with 1-year followup. Ann Surg 2001;233(6):725-732.

19. Autschbach R, Onnasch JF, Falk V, Walther T, Kruger M, Schilling LO, et al. The Leipzig experience with robotic valve surgery. J Card Surg 2000;15(1):82-87.

20. Melfi FM, Menconi GF, Mariani AM, Angeletti CA. Early experience with robotic technology for thoracoscopic surgery. Eur J Cardiothorac Surg 2002;21(5):864-868. 
21. Yoshino I, Hashizume M, Shimada M, Tomikawa M, Tomiyasu M, Suemitsu R, et al. Thoracoscopic thymomectomy with the Da Vinci computer-enhanced surgical system. J Thorac Cardiovasc Surg 2001;122(4):783-785.

22. Ruurda JP, Hanlo PW, Hennipman A, Broeders IA. Robotassisted thoracoscopic resection of a benign mediastinal neurogenic tumor: technical note. Neurosurgery 2003;52 (2):462-464.

23. Abbou CC, Hoznek A, Salomon L, Olsson LE, Lobontiu A, Saint F, et al. Laparoscopic radical prostatectomy with a remote controlled robot. J Urol 2001;165(6 Pt 1):19641966.

24. Binder J, Kramer W. Robotically-assisted laparoscopic radical prostatectomy. BJU Int 2001;87(4):408-410.

25. Pasticier G, Rietbergen JB, Guillonneau B, Fromont G, Menon M, Vallancien G. Robotically assisted laparoscopic radical prostatectomy: feasibility study in men. Eur Urol 2001;40(1):70-74.

26. Young JA, Chapman WH, III, Kim VB, Albrecht RJ, Ng PC, Nifong LW, et al. Robotic-assisted adrenalectomy for adrenal incidentaloma: case and review of the technique. Surg Laparosc Endosc Percutan Tech 2002;12(2):126-130.

27. Horgan S, Vanuno D, Sileri P, Cicalese L, Benedetti E. Robotic-assisted laparoscopic donor nephrectomy for kidney transplantation. Transplantation 2002;73(9):14741479.

28. Hoznek A, Zaki SK, Samadi DB, Salomon L, Lobontiu A, Lang P, et al. Robotic assisted kidney transplantation: an initial experience. J Urol 2002;167(4):1604-1606.

29. Walsh PC. Radical retropubic prostatectomy with reduced morbidity: an anatomic approach. NCI Monogr 1988;(7): 133-137.

30. Walsh PC, Partin AW. Treatment of early stage prostate cancer: radical prostatectomy. Important Adv Oncol 1994; 211-223.

31. Walsh PC. Radical prostatectomy: a procedure in evolution. Semin Oncol 1994;21(5):662-671

32. Walsh PC. Anatomic radical prostatectomy: evolution of the surgical technique. J Urol 1998;160(6 Pt 2):24182424.

33. Walsh PC, Lepor H, Eggleston JC. Radical prostatectomy with preservation of sexual function: anatomical and pathological considerations. Prostate 1983;4(5):473-485.

34. Reiner WG, Walsh PC. An anatomical approach to the surgical management of the dorsal vein and Santorini's plexus during radical retropubic surgery. J Urol 1979;121(2):198200.

35. Schuessler WW, Schulam PG, Clayman RV, Kavoussi LR. Laparoscopic radical prostatectomy: initial short-term experience. Urology 1997;50(6):854-857.

36. Guillonneau B, Vallancien G. Laparoscopic radical prostatectomy: initial experience and preliminary assessment after 65 operations. Prostate 1999;39(1):71-75.

37. Guillonneau B, Vallancien G. Laparoscopic radical prostatectomy: the Montsouris technique. J Urol 2000;163(6): 1643-1649

38. Abbou CC, Salomon L, Hoznek A, Antiphon P, Cicco A, Saint F, et al. Laparoscopic radical prostatectomy: preliminary results. Urology 2000;55(5):630-634.
39. Rassweiler J, Seemann O, Schulze M, Teber D, Hatzinger M, Frede T. Laparoscopic versus open radical prostatectomy: a comparative study at a single institution. J Urol 2003;169(5):1689-1693.

40. Brown JA, Garlitz C, Gomella LG, Hubosky SG, Diamond SM, McGinnis D, et al. Pathologic comparison of laparoscopic versus open radical retropubic prostatectomy specimens. Urology 2003;62(3):481-486.

41. Mitka M. Laparascopic prostate surgery suggested. JAMA 2001;286(18):2224

42. Anastasiadis AG, Salomon L, Katz R, Hoznek A, Chopin $\mathrm{D}$, Abbou CC. Radical retropubic versus laparoscopic prostatectomy: a prospective comparison of functional outcome. Urology 2003;62(2):292-297.

$42^{\text {bis }}$ Pascual Piédrola JI, Grasa Lanau V, Cuesta Alcalá JA, Aldave Villanueva J, Ponz González M, Ipiens Aznar A. Prostatectomía radical laparoscópica. ¿Sólo en Centros de Excelencia?. Actas Urol Esp 2005; 10:927-933

43. Martina GR, Giumelli P, Scuzzarella S, Remotti M, Caruso G, Lovisolo J. Laparoscopic extraperitoneal radical prostatectomy-learning curve of a laparoscopy-naive urologist in a community hospital. Urology 2005;65(5): 959-963.

44. Stolzenburg JU, Truss MC, Bekos A, Do M, Rabenalt R, Stief CG, et al. Does the extraperitoneal laparoscopic approach improve the outcome of radical prostatectomy? Curr Urol Rep 2004;5(2):115-122.

45. Menon M, Tewari A, Baize B, Guillonneau B, Vallancien G. Prospective comparison of radical retropubic prostatectomy and robot-assisted anatomic prostatectomy: the Vattikuti Urology Institute experience. Urology 2002;60 (5):864-868.

46. Tewari A, Srivasatava A, Menon M. A prospective comparison of radical retropubic and robot-assisted prostatectomy: experience in one institution. BJU Int 2003;92(3): 205-210.

47. Bentas W, Wolfram M, Jones J, Brautigam R, Kramer W, Binder J. Robotic technology and the translation of open radical prostatectomy to laparoscopy: the early Frankfurt experience with robotic radical prostatectomy and one year follow-up. Eur Urol 2003;44(2):175-181.

48. Ahlering TE, Woo D, Eichel L, Lee DI, Edwards R, Skarecky DW. Robot-assisted versus open radical prostatectomy: a comparison of one surgeon's outcomes. Urology 2004;63 (5):819-822.

49. Villavicencio H. Tecnología de futuro: Cirugía robótica Da Vinci. Actas Urol Esp 2005; 29(10): 919-921

50. Satava RM. Future trends in the design and application of surgical robots. Semin Laparosc Surg 2004;11(2):129135 .
Dr. H. Villavicencio Mavrich
Servicio de Urología
Fundació Puigvert
Cartagena, 340-350
08025 Barcelona

(Trabajo recibido el 2 de enero 2006) 